\title{
Automatic Download Design of STC MCU Based on CH340G
}

\author{
Zilong $\mathrm{Ma}^{\mathrm{a}}$, Xiang $\mathrm{Li}^{\mathrm{b},{ }^{*}}$ \\ Guangxi Technological College of Machinery and Electricity, Nanning 530007, China \\ amazilong@163.com, bxiangzi21th@hotmail.com
}

\begin{abstract}
This paper presents a design of automatic STC MCU program downloader based on CH340G USB serial converter chip. The user through the host computer to achieve the STC 5V/3.3V MCU to erase, program, check and encrypt. The download device is not required for MCU control, and has the advantages of simple circuit, low price, stable function, fast download speed, no need of cold starting etc.
\end{abstract}

Keywords: STC MCU; Automatic; Downloader; UART.

\section{Introduction}

STC MCU is currently the most widely used and most popular of the 51 series MCU. User written code in the download into the STC MCU FLASH, the need for a computer serial interface with the STC MCU ISP monitor program to shake hands to achieve the program download function. Now, with the development of computer technology, whether it is a desktop or notebook computer, rarely leaving the serial interface to the microcontroller of this program download, debugging inconvenience, so it promotes the development of USB downloader. In addition, STC Series MCU ISP monitor program is running on the power up, if you want to download the program code, you need to restart the MCU, which is the first to disconnect the power supply, and then on the power. The usual practice is in the process of continuous pressing circuit power switch device, which is what we usually say STC microcontroller cold start. Cold start of the download mode is not only easy to damage the switch device, it is not easy to program compiler software KEIL with the STC single chip, reducing the product development efficiency.

\section{Hardware Design}

\subsection{STC MCU Introduction.}

STC MCU Limited is the Chinese mainland local MCU leader, the company has launched STC89C/S, 51xx/52xx, STC12C, STC15F and many other series, has the advantages of low price, low power consumption, good performance, not declassified, antistatic and strong anti-interference. The STC MCU retains all features of the standard 80C51.Its on-chip resources are very rich, and have built-in rewritable FLASH memory. STC12C5A60S2 as a typical representative of STC MCU, and its main features are: 60K bytes of FLASH, 1000 times Endurance, 1280 bytes RAM, 1T instruction cycle, five IO ports, and the EEPROM function. In addition, the STC12C5A60S2 has two extra I/O ports (P4 and P5), a 10-sources, 4-priority-level interrupt structure, 10-bit ADC, two UARTs, on-chip crystal oscillator, a 2-channel PCA and PWM, SPI, a one-time enabled Watchdog Timer.

In-System-Programming (ISP) and In-Application-Programming (IAP) support the users to upgrade the program and data in system. ISP allows the user to download new code without removing the microcontroller form the actual end product; IAP means that the device can write non-volatile data in Flash memory while the application program is running. ISP will not damage the chip pin, while the download speed quickly, thus reducing design time, reduce development costs and time to market for the product, greatly improve work efficiency [1]. The programmer is the use of FT232, PL2303, CP2102 control chip, but it is of high cost, stability. This paper proposes a ISP programmer design scheme based on domestic CH340G chip as the main body, and can automatically download, without cold start, after repeated testing, STC Series MCU function stability, outstanding performance. 


\subsection{CH340G Introduction.}

CH340 is a USB bus convert chip and it can realize USB convert to serial interface, USB convert to IrDA infrared or USB convert to printer interface. In serial interface mode, $\mathrm{CH} 340$ supplies common MODEM liaison signal, used to enlarge asynchronous serial interface of computer or upgrade the common serial device to USB bus directly. $\mathrm{CH} 340 \mathrm{G}$ has the following characteristics [2]:

(1) Full speed USB device interface, conforms to USB Specification Version 2.0, only needs crystal and capacitance external.

(2) Hardware full duplex serial interface, set transceiver buffer, supports communication baud rate varies from $50 \mathrm{bps}$ to $2 \mathrm{Mbps}$.

(3) Supports common MODEM liaison signal RTS, DTR, DCD, RI, DSR and CTS.

(4) Through adding level converter equipment to supply RS232, RS485, RS422 and other interface.

(5) Supports IrDA criterion SIR infrared communication, supports baud rate varies from $2400 \mathrm{bps}$ to $115200 \mathrm{bps}$.

(6) Support 5V and 3.3V source voltage.

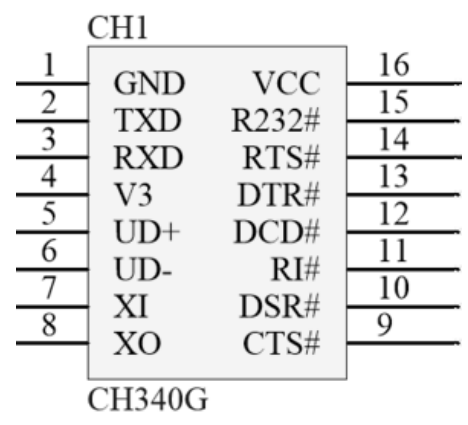

Fig.1 CH340G Package

CH340G chip set up power up reset circuit internal. CH340G chip is shown in Fig.1, including 16 pins, each pin functions as shown in Table 1.

Table 1 CH340G Pin Description

\begin{tabular}{ccc}
\hline Pin No. & Pin Name & Pin Description \\
\hline 1 & GND & Public ground, ground connection for USB \\
2 & TXD & Serial data output \\
3 & RXD & Serial data input \\
4 & V3 & connects of VCC to input outside power while 3.3V \\
5 & UD+ & Directly connect to D+ data wire of USB bus \\
6 & UD- & Directly connect to D- data wire of USB bus \\
7 & XI & Crystal oscillator input \\
8 & XO & Crystal oscillator output \\
9 & CTS\# & MODEM liaison input signal, clear sending \\
10 & DSR\# & MODEM liaison input signal, data equipment is ready \\
11 & RI\# & MODEM liaison input signal, oscillate ring to prompt \\
12 & DCD\# & MODEM liaison input signal, carrier wave detection \\
13 & DTR\# & MODEM liaison output signal, Data endpoint is ready \\
14 & RTS\# & MODEM contact output signal, request to send \\
15 & R232 & Assistant RS232 enable, active with high \\
16 & VCC & Positive power input port \\
\hline
\end{tabular}




\subsection{STC MCU Program Download Process.}

STC all of the microcontroller has been integrated ISP module, can be online programming, through the verification of the data flow directly into the user program area, complete the user program download action [3]. ISP execution as follows:

The PC through the download software (recommended by STC free software) ISP download a command stream, the MCU control start (download here needs some cold start, the key of this design is to realize the automatic start here), after starting the first run the factory default ISP monitoring program. Answer a handshake when the ISP monitoring program detects the RXD pin ISP command stream. After the handshake is successful, the software will download the user code to send to the microcontroller, ISP will receive the user program writes the user program area. Receives the data transfer to complete the sign, the monolithic computer then executes the reset instruction, causes the program pointer PC to point to the user program area the initial address, completes the downloading process. If the MCU starts, ISP monitoring program failed to detect the presence of RXD download command stream, directly to the program pointer to the start address of the user program area, which is not directly perform the download operation run user programs.

\subsection{Programmer circuit.}

Using USB as the $\mathrm{CH} 340 \mathrm{G}$ UART (serial port) control chip, the external circuit wiring as shown in Fig.2.

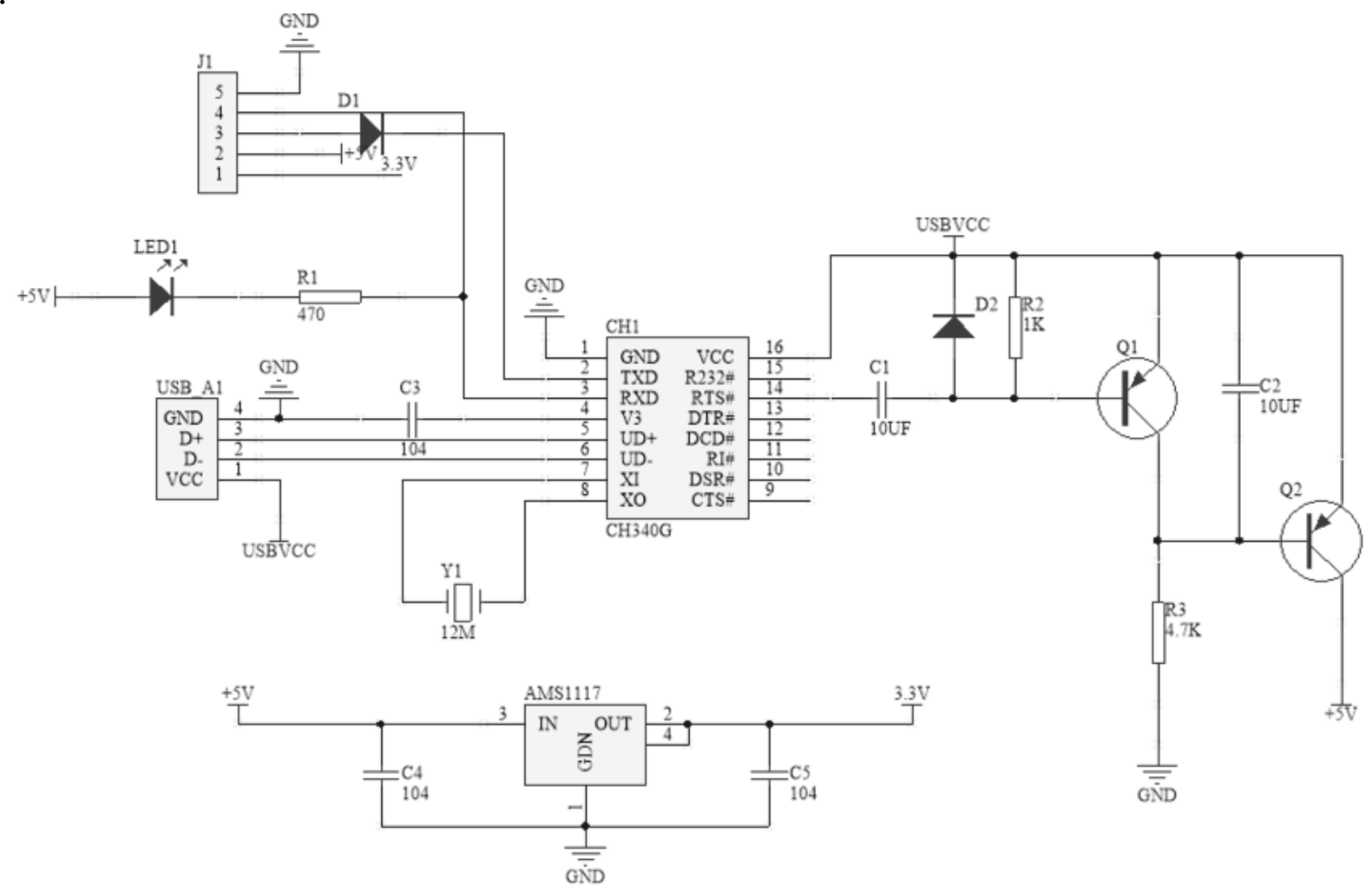

Fig. 2 Circuit of STC automatic download based on CH340G

Support 5V STC and 3.3V Series MCU program automatically download the circuit, including the USB interface circuit, $\mathrm{CH} 340 \mathrm{G}$ serial communication circuit, automatic download processing circuit, $3.3 \mathrm{~V}$ voltage generation circuit and download interface circuit. USB and serial interface circuit to complete the computer chip data communication connections and system power supply voltage of $5 \mathrm{~V}$, the transfer is completed $\mathrm{CH} 340 \mathrm{G}$ serial chip microcontroller download data and generate a falling edge signal on the RTS \# pin, automatic download processing circuit provided by RTS\# trigger signal generating extremely short delay pulse signal a low level of $5 \mathrm{~V}, 3.3 \mathrm{~V}$ voltage generating circuit by the output 3.3V voltage regulator chip AMS1117-3V3 containing a very low level of short delay pulse signal, interface circuit to STC download the microcontroller provides interface signals $5 \mathrm{~V}$ and $3.3 \mathrm{~V}$, and TX, RX, GND, etc. required to download the program. According to STC microcontroller chip supply voltage $5 \mathrm{~V}$ or $3.3 \mathrm{~V}$ asked to choose in the download interface circuit, the voltage of the power supply in the role of RTS\# signal, resulting in a very short low-level signals, and after pulled is high 
level, the equivalent of STC microcontroller broke off, and then power is restored, then equivalent to the STC microcontroller restart, so to achieve a single-chip STC automatic download function.

\section{System test}

Download production is successful, need to install the $\mathrm{CH} 340 \mathrm{G}$ drivers on PC, can be downloaded from the official website of Nanjing qinheng Electronics Co., ltd. After installing the driver, plug download, which can be found in the new virtual serial port in the PC device manager, remember its number, such as COM3. After these preparations are ready, you need to STC Company's official website to download the program software STC-ISP, STC-ISP is designed for STC microcontroller download software, all of the chips are common.

The steps of the test is, first we choose the development board based on STC12C5A60S2 chip are used widely, and the development board of the VCC, RXD, TXD, GND of the four connection port respectively and download the VCC, TXD, RXD, GND corresponding line connected by Dupont Line. It should be noted that the microcontroller TXD, RXD two lines to be connected with the TXD, RXD data line of the download device. Then plug the download to PC USB port, and then open the STC-ISP software, a specific type of selected MCU microcontroller in the interface (here STC12C5A60S2), leading to download the user program (generally HEX file), select the virtual serial port, and finally click the download button, the program can be automatically downloaded to the STC single chip FLASH, without cold start.

After repeated testing and verification, the design of this paper to support the STC full series MCU automatic programming download, no need for cold start, the function is normal, the performance is reliable.

\section{Conclusion}

In this paper, a design of automatic download for STC MCU circuit based on CH340G, tested by practice, it has a simple circuit, reliable, excellent performance, low cost, download speed, without cold start characteristics.

\section{References}

[1] Tong Huang, Sifei Shao. A Programmer Design Based on CH340T for STC89C52RC. Electronic test. Vol. 12 (2013), p. 16-17.

[2] Information on: www.stcmcu.com.

[3] Zhe Weng. A Design for STC MCU Program Downloader. Joumal of Xi'an University of Arts \& Science (Nat Sci Ed), Vol. 13 (2010) No. 3, p. 47-50. 